\title{
Late-phase immune responses limiting oocyst survival are independent of TEP1 function yet display strain specific differences in Anopheles gambiae
}

\author{
Hyeogsun Kwon, Benjamin R. Arends and Ryan C. Smith ${ }^{*}$
}

\begin{abstract}
Background: There is emerging evidence that mosquito anti-Plasmodium immunity is multimodal with distinct mechanisms for killing malaria parasites at either the ookinete or oocyst stages. Early-phase responses targeting the ookinete require complement-like components circulating in the mosquito hemolymph that result in TEP1-mediated lysis or melanization. Additional responses mediated by the LL3 and STAT pathways limit oocyst survival through unknown mechanisms that require mosquito hemocyte function. While previous experiments argue that these mechanisms of parasite killing are independent, the transient nature of gene-silencing has rendered these experiments inconclusive. To address this issue, we outline experiments using a TALEN-derived TEP1 mutant line to examine the role of TEP1 in the Anopheles gambiae late-phase immune response.
\end{abstract}

Results: Despite higher early oocyst numbers in the TEP1 mutant line, no differences in oocyst survival were observed when compared to control mosquitoes, suggesting that TEP1 function is independent of the late-phase immune response. To further validate this phenotype in the TEP1 mutant, oocyst survival was evaluated in the TEP1 mutant background by silencing either LL3 or STAT-A. Surprisingly, only STAT-A silenced mosquitoes were able to reconstitute the late-phase immune phenotype increasing oocyst survival in the TEP1 mutant line. Additional experiments highlight significant differences in LL3 expression in the M/S hybrid genetic background of the TEP1 mutant line compared to that of the Keele strain ( $M$ form) of An. gambiae, and demonstrate that LL3 is not required for granulocyte differentiation in the M/S hybrid G3 genetic background in response to malaria parasite infection.

Conclusions: Through the combination of genetic experiments utilizing genetic mutants and reverse genetic approaches, new information has emerged regarding the mechanisms of mosquito late-phase immunity. When combined with previously published experiments, the body of evidence argues that Plasmodium oocyst survival is TEP1 independent, thus establishing that the mechanisms of early- and late-phase immunity are distinct. Moreover, we identify that the known components that mediate oocyst survival are susceptible to strain-specific differences depending on their genetic background and provide further evidence that the signals that promote hemocyte differentiation are required to limit oocyst survival. Together, this study provides new insights into the mechanisms of oocyst killing and the importance of genetics in shaping mosquito vector competence.

Keywords: Anopheles gambiae, Innate immunity, Hemocytes, Plasmodium berghei, Late-phase immunity, TEP1, TALENs, RNAi, LL3, STAT-A, Oocyst survival, Mosquito genetics

\footnotetext{
* Correspondence: smithr@iastate.edu

Department of Entomology, lowa State University, Ames, lowa 50011, USA
} 


\section{Background}

Malaria is a devastating disease caused by Plasmodium parasites that results in approximately half a million deaths per year, predominantly in sub-Saharan Africa [1]. Transmitted through the bite of an infected anopheline mosquito, the interactions between the parasite and its mosquito host are major determinants of vector competence [2]. Recognition of Plasmodium parasites by the mosquito immune system is believed to be an integral step in defining vector competence, with only those parasites able to successfully evade immune recognition are capable of transmission [3, 4]. As such, a great deal of effort has been invested to better understand the mechanisms of parasite killing in the mosquito host $[2,5]$.

Evidence suggests that the mosquito innate immune system has a significant role in killing malaria parasites at the ookinete [6-8] and oocyst stages $[9,10]$, yet our understanding of the anti-Plasmodium immune responses that limit these respective parasite stages remains incomplete. Ookinete invasion triggers epithelial nitration responses that enable parasite recognition by the mosquito complement-like system [11], promoting the deposition of thioester-containing protein 1 (TEP1) and other proteins on the ookinete surface that ultimately lead to parasite lysis or melanization [6-8, 12-14]. In addition, for those parasites able to evade mosquito complement, recent data argues that a second "late-phase" response limits oocyst survival $[9,10]$. Mediated by the yet unknown effects of hemocyte differentiation, the transcription factors LPSinduced TNF-alpha factor (LITAF)-like 3 (LL3) and signal transducer and activator of transcription A (STAT-A) are integral to these responses $[5,9,10]$. Furthermore, evidence suggests that the late-phase response is independent of TEP1 function [10].

However, further validation is required to confirm that the late-phase response is independent of TEP1 and mosquito complement function. Previous studies have demonstrated that the loss of TEP1 by RNAi did not influence oocyst survival [10], yet due to the temporal and spatial limitations of gene silencing in mosquitoes that may have allowed TEP1 to return to functional levels, the potential role of TEP1 on oocyst survival has yet to be fully explored. In an effort to follow up with these studies by Smith et al. [10], we examined the late-phase immune response in a mutant-TEP1 An. gambiae background to elucidate roles of TEP1 and complement-like function in determining oocyst survival.

Here we provide further evidence that oocyst survival is independent of TEP1 function and in the process uncover new insights into differences in gene regulation across mosquito strains that influence Plasmodium oocyst survival. Together, these data argue that late-phase immune responses are distinct from those that eliminate Plasmodium ookinetes and provide evidence that the genetic complexity of natural An. gambiae populations may significantly influence mosquito immune responses in geographically distinct regions of endemic malaria transmission.

\section{Methods \\ Mosquito rearing}

Anopheles gambiae mosquitoes of the Keele strain [15], or TALEN-derived TEP1 mutant and parental control $\mathrm{X} 1$ lines derived from a laboratory $A n$. gambiae G3 strain [16] were reared at $27{ }^{\circ} \mathrm{C}$ and $80 \%$ relative humidity, with a $14 / 10 \mathrm{~h}$ day/night cycle. Larvae were fed on fish food flakes (Tetramin, Tetra) and adult mosquitoes were maintained on $10 \%$ sucrose solution.

\section{Plasmodium infection}

Female Swiss Webster mice were infected with a mCherry strain of $P$. berghei as previously described [10]. Naïve An. gambiae mosquitoes (4-6 day old) from either the control X1, TEP1 mutant, or Keele lines were challenged with an infected anesthetized mouse displaying 2-3 exflagellation centers when evaluated under a compound microscope with a $10 \times$ objective. Infected mosquitoes were maintained at $19{ }^{\circ} \mathrm{C}$ until oocyst numbers were examined in individual dissected midguts at day 2 and day 8 by fluorescent microscopy (Nikon Eclipse 50i, Nikon) using the same cohort of mosquitoes as previously [10].

\section{RNAi and qRT-PCR}

dsRNA synthesis was performed as previously described for GFP, STAT-A, and LL3 [10, 17]. Briefly, gene-specific primers flanked with T7 promoter sequences (described in Additional file 1: Table S1) were used to PCR amplify using plasmid templates for GFP, STAT-A, and LL3. Resulting T7 DNA products were used as a template for dsRNA synthesis using the MEGAscript RNAi kit (Life Technologies) according to the manufacturer's instructions. All dsRNA was diluted in nuclease free water to $3 \mu \mathrm{g} / \mu \mathrm{l}$. Naïve control X1 and TEP1 mutant mosquitoes (3- to 4-day old) were cold anesthetized and injected in the thorax with $\sim 200 \mathrm{ng}$ dsRNA. To evaluate the effects of gene-silencing on oocyst development, mosquitoes were challenged with $P$. berghei at 2 days post-injection. Oocyst numbers were examined at 2 and 8 days postinfection using the same cohort of mosquitoes as described above.

To measure the effects of gene-silencing, total RNA was isolated from $\sim 15$ whole mosquitoes, 2 days post-injection using TRIzol (Thermo Fisher Scientific, Waltham, MA, USA). The isolated RNA was purified with the RNA Clean \& Concentrator Kit (Zymo Research, Orange, CA, USA) and quantified using a NanoDrop spectrophotometer. cDNA was synthesized using $2 \mu \mathrm{g}$ of total RNA using the 
RevertAid First Strand cDNA Synthesis Kit (Thermo Fisher Scientific) according to the manufacturer's protocol. Relative gene expression was analyzed using PowerUp ${ }^{\mathrm{Tm}} \mathrm{SYBR}^{\odot}$ Green Master Mix (Thermo Fisher Scientific), a 1:5 dilution of cDNA template, and $250 \mathrm{nM}$ of gene-specific primer (Additional file 1: Table S1). Ribosomal protein S7 transcript was used as a reference to determine relative transcript levels as previously [17]. The thermal cycling conditions for PCR were: $95{ }^{\circ} \mathrm{C}$ for $10 \mathrm{~min}, 40$ cycles with $95{ }^{\circ} \mathrm{C}$ for $15 \mathrm{~s}$ and $63{ }^{\circ} \mathrm{C}$ for $60 \mathrm{~s}$, and PCR was run on a QuantStudio 3 (Thermo Fisher Scientific). A comparative $\mathrm{C}_{\mathrm{T}}\left(2^{-\Delta \Delta \mathrm{Ct}}\right)$ method was employed to evaluate relative transcript abundance for each transcript [18].

To examine differences in relative gene expression of $L L 3$ and STAT-A in the different mosquito strains, total RNA was isolated from 20 midguts of Keele, X1 and G3 mosquitoes at non-blood fed and $24 \mathrm{~h} P$. berghei infection. The cDNA synthesis and qRT-PCR was performed as described above using $1 \mu \mathrm{g}$ of total RNA.

\section{Identification of molecular forms by PCR-RFLP}

To determine whether colonized lab strains of Anopheles gambiae used in the study were M- (now referred to as An. coluzzii [19]) or S-form, genomic DNA was prepared from individual adult female mosquitoes using a Marriot DNA extraction protocol for the X1, TEP1-Mut, G3, Keele and Ngousso strains [20]. Individual mosquito DNA samples were used as a template for PCR-RFLP analysis to distinguish between $\mathrm{M}$ and $\mathrm{S}$ molecular forms as previously described [21]. Following PCR amplification, samples were purified using a DNA Clean and Concentrator Kit (Zymo Research), then digested with FastDigest Hhal (Thermo Fisher Scientific) overnight at $37{ }^{\circ} \mathrm{C}$. DNA fragments were visualized by gel electrophoresis using a 1.5\% agarose gel. Mosquito samples were scored as being M-form (367 bp), S-form (257 and $110 \mathrm{bp}$ ), or as M/S hybrid species (367, 257 and $110 \mathrm{bp}$ ) through restriction fragment length polymorphisms (RFLP). Approximately 10 mosquitoes were analyzed from each strain, displaying a consistent RFLP pattern across all samples.

\section{Hemolymph perfusion and hemocyte counting}

Following dsRNA-mediated gene-silencing, mosquitoes from either the G3 or Keele strains were perfused 4 days after $P$. berghei infection as previously described [10, 22]. The collected hemolymph was placed in a Neubauer Improved hemocytometer and individual hemocytes were distinguished by morphology using light microscopy [10] More than 200 cells per individual mosquito were analyzed to determine the proportion of granulocytes out of the total cell population [10].

\section{Statistical analysis}

All Plasmodium infection experiments were performed in three or more independent experiments. Pooled data was analyzed using either a Mann-Whitney or KruskalWallis with a Dunn's post-hoc test. Gene expression data from at least three independent biological replicates were analyzed using an unpaired (student's) t-test for individual comparisons or by a one-way ANOVA with a Tukey post-hoc test for multiple comparisons. Granulocyte percentages were analyzed by Kruskal-Wallis with a Dunn's post-hoc test. All statistical analysis and graphing were performed with GraphPad Prism (GraphPad Software Inc., CA, USA).

\section{Results}

To address potential role of TEP1 in limiting oocyst survival, we utilized a previously characterized TALENderived TEP1 mutant line ( $\Delta$ ct1) [16] to examine both early and late oocyst numbers following $P$. berghei infection as previously described $[9,10]$. Using the X1 parental strain from which the TEP1 mutant line was originally derived as a control, oocyst numbers were examined at day 2 and day 8 following infection with fluorescent mCherry $P$. berghei parasites. Oocyst numbers significantly decreased $(U=1307, Z=4.32$, $P<0.0001)$ in the control-X1 line between day 2 and day 8 (Fig. 1a) as previously described [9, 10, 23]. When similar experiments were performed using a TEP1 mutant line, oocyst numbers were also significantly reduced ( $U=1531, Z=4.98, P<0.0001$; Fig. $1 \mathrm{~b}$ ), arguing that TEP1 function is not required for late-phase immune responses limiting oocyst survival as previously suggested [10].

To further demonstrate that the immune responses limiting oocyst survival are independent of TEP1 function, we performed gene-silencing experiments in the TEP1 mutant background to target STAT-A and LL3, known mediators of late-phase immunity $[9,10]$. Following STAT- $A$ silencing in the TEP1 mutant line $(t=4.946$, $P=0.0078$; Additional file 2: Figure S1), Day 2 oocyst numbers were similar between STAT-A and control (GFP) treatments (Fig. 2). A significant loss in oocyst numbers was measured in the period between day 2 and day 8 for the dsGFP control mosquitoes $\left(\chi^{2}=20.91, d f=3\right.$, $P=0.0001)$, one that was abrogated upon STAT-A silencing (Fig. 2). This is consistent with previous reports that argue that STAT-A influences oocyst survival $[9,10]$, and provides additional support that the late-phase immune response remains intact in the TEP1 mutant background.

The effects of LL3 silencing in the TEP1 mutant background were examined in similar experiments. To our surprise, no difference in oocyst numbers was detected in LL3-silenced mosquitoes when compared to dsGFP controls (Fig. 3a) despite a significant reduction in $L L 3$ gene expression $(t=7.152, P=0.002$; Additional file 2: Figure S1). 

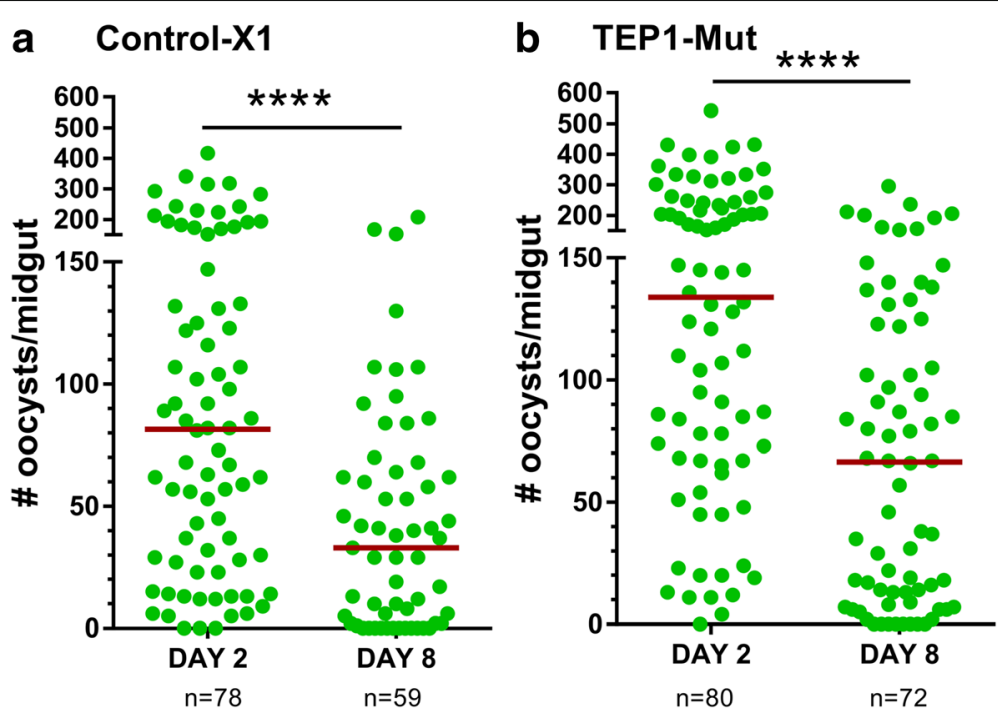

Fig. 1 Late-phase immunity is intact in both control and TEP1 mutant lines. Oocyst numbers in the midgut were determined in control-X1 and TEP1 mutant lines at 2 and 8 days post- $P$. berghei infection. Oocyst survival in both control-X1 (a) and TEP1 mutant (b) lines is reduced between day 2 and day 8 as measure by oocyst numbers. For both experiments, each dot represents the number of oocysts on an individual midgut. Oocyst numbers were pooled from three independent experiments, with the median indicated by the horizontal red line. Oocyst numbers were analyzed using a Mann-Whitney test. Asterisks denote significance $\left(^{* * *} P<0.0001\right)$

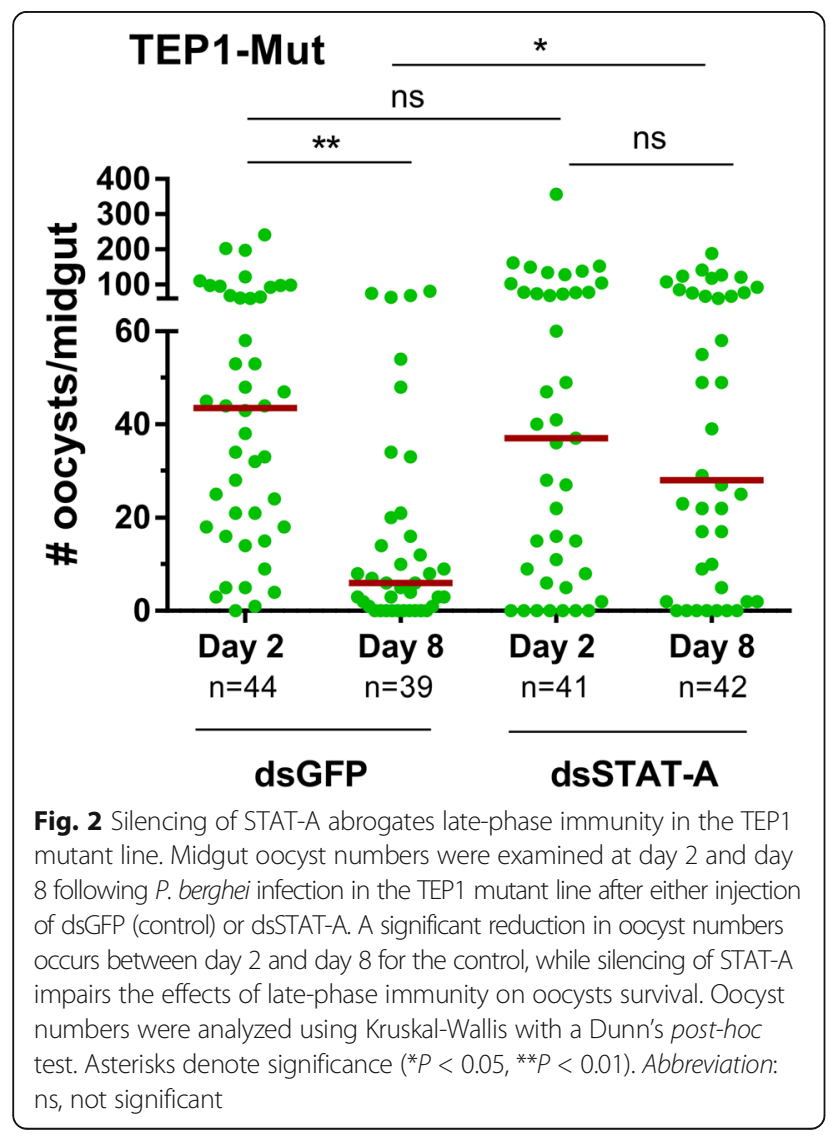

Moreover, $L L 3$-silencing did not increase oocyst survival in the mutant TEP1 line, contrary to previous results examined in the Keele strain of An. gambiae [10, 17]. However, when these RNAi experiments were again repeated in the wild-type Keele strain, $L L 3$-silencing caused a significant increase $(U=1375, Z=2.22, P=0.025)$ in oocyst survival in agreement with previous experiments (Fig. 3b) [10, 17].

These contrasting results led us to examine the genetic backgrounds of the mosquito strains in question to determine if genetic differences between the lines may account for these discrepancies in $L L 3$ gene function. The TEP1 mutant line and the parental X1 line were originally derived from a laboratory G3 colony [16], while the Keele strain was developed from interbreeding four African-derived laboratory colonies $[15,24]$. To determine potential differences in these mosquito strains, we examined individual mosquitoes from each strain for the $\mathrm{M}$-form or $\mathrm{S}$ form rDNA markers present on the $\mathrm{X}$ chromosome that have recently been used to distinguish between An. coluzzii (M-form) and An. gambiae (s.s.) (S-form). All individual mosquito samples collected from each strain displayed consistent patterns when analyzed by a PCR-RFLP assay to [21], confirming that the X1, TEP1 mutant, and G3 lines are M/S hybrid form (Fig. 4a). In contrast, the Keele and Ngousso strains are M-form derived (Fig. 4a). These results argue that the Keele strain be reclassified as An. coluzzii according to Coetzee et al., although recent reports have pushed that the Keele strain maintain its reference of $A n$. gambiae (s.s.) [24]. 

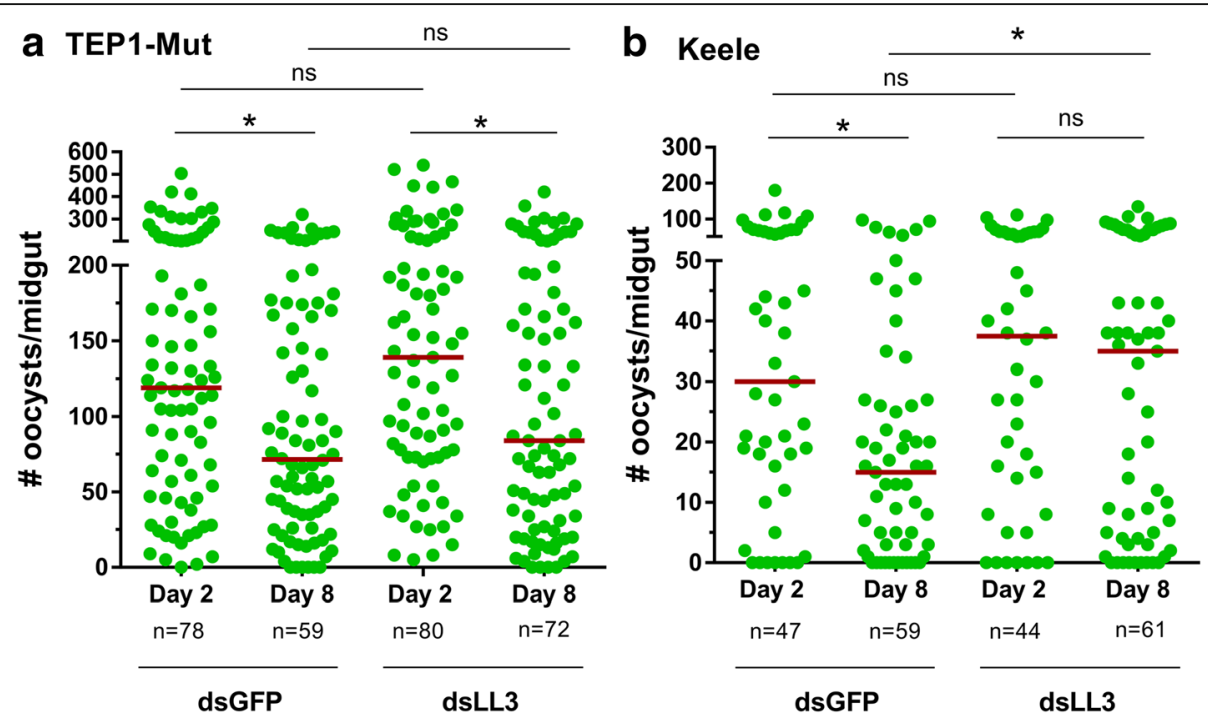

Fig. 3 The effects of LL3-silencing on Plasmodium survival vary between mosquito strains. Oocyst numbers were examined in the TEP1 mutant line and Keele strain after injection of dsGFP (control) or dsLL3 at day 2 and day 8 following P. berghei infection. Significant losses in parasite numbers were detected for both the dsGFP-treated control and LL3-silenced mosquitoes at day 8 (a), while LL3 silencing did not influence on early oocyst development at day 2 in the TEP1-Mut line. In contrast, oocyst survival is increased following LL3-silencing in the Keele stain (b). Oocyst numbers were analyzed by Kruskal-Wallis with a Dunn's post-hoc test (a) or by Mann-Whitney analysis $(\mathbf{b})$. Asterisks denote significance $\left({ }^{*} P<0.05\right)$. Abbreviation: ns, not significant

Given the differences in LL3 function between the TEP1 mutant and the Keele strain (Fig. 3), genetic divergence between the G3-derived and the Keele strains may account for the differing RNAi phenotypes on oocyst survival. Preliminary in silico analysis did not find allelic polymorphisms in LL3 or STAT-A between M- and S-forms. To explore potential differences in gene regulation, we examined $L L 3$ gene expression in the X1, G3, and Keele strains in naive mosquitoes as well as $24 \mathrm{~h}$ post-infection with $P$. berghei. Under naïve conditions, basal levels of $L L 3$ expression were highest in the Keele strain, yet significant differences were only found for the X1 strain when compared to G3 and Keele (Additional file 3: Figure S2). Similar to previous experiments [10, 17], LL3 was significantly upregulated across all strains in response to $P$. berghei infection $(t=17.06$, $P<0.0001$ in X1, $t=7.394, P=0.0018$ in G3, $t=11.02$, $P=0.0004$ in Keele; Additional file 3: Figure S2), yet the levels of $L L 3$ induction in the Keele strain were $~ 3-$ to 7 fold higher than the other lines after feeding on an infected blood meal $\left(F_{(2,6)}=42.62, P=0.0002\right.$; Fig. $\left.4 \mathrm{~b}\right)$. In contrast, similar analysis of STAT-A expression did not find significant differences in gene expression across the X1, G3, and Keele strains under naïve and infected treatments (Additional file 3: Figure S2; Fig. 4c), yet display significant up regulation in the Keele strain to $P$. berghei infection $(t=3.095, P=0.036)$. Together, these results argue that the differential regulation of $L L 3$, but not STAT-A, may explain the differences in latephase immune function between mosquito strains.

With previously described roles on hemocyte differentiation in An. gambiae [10, 25], we examined the effects of LL3 and STAT-A silencing on hemocyte differentiation in both the G3 and Keele strains. Following parasite infection, the proportion of granulocytes were comparable in control and $L L 3$-silenced mosquitoes in the G3 strain (Fig. 4d), while granulocyte differentiation was abrogated following LL3-silencing in the Keele strain $\left(\chi^{2}=17.87, d f=2, P=0.0001\right.$, Fig. 4e) similar to previous observations [10]. In contrast, the proportion of granulocytes were reduced in STAT-Asilenced mosquitoes for both the G3 $\left(\chi^{2}=35.26, d f=2\right.$, $P<0.0001)$ and Keele strains $\left(\chi^{2}=17.87, d f=2\right.$, $P=0.0001$,Fig. $4 \mathrm{~d}, \mathrm{e})$. These data suggest that LL3 influences hemocyte differentiation only in the Keele strain, while the involvement of the STAT pathway on hemocyte differentiation is conserved in both strains. These findings represent the first description of strain-specific differences in the late-phase response and the mechanisms that limit oocyst survival.

\section{Discussion}

Mosquito innate immune responses are integral determinants of vector competence and subsequent mosquito-borne disease transmission. Evidence suggests that multiple waves of the mosquito immune response limit malaria parasite development, with specific responses that target the ookinete and oocyst stages of Plasmodium development $[2,5]$.

Previous experiments have suggested that the mechanisms that define these "early" and "late" responses are independent [10], yet limitations in gene-silencing techniques have left this question unresolved.

Taking advantage of an existing TALEN-derived mutant TEP1 line [16], our experiments provide compelling 

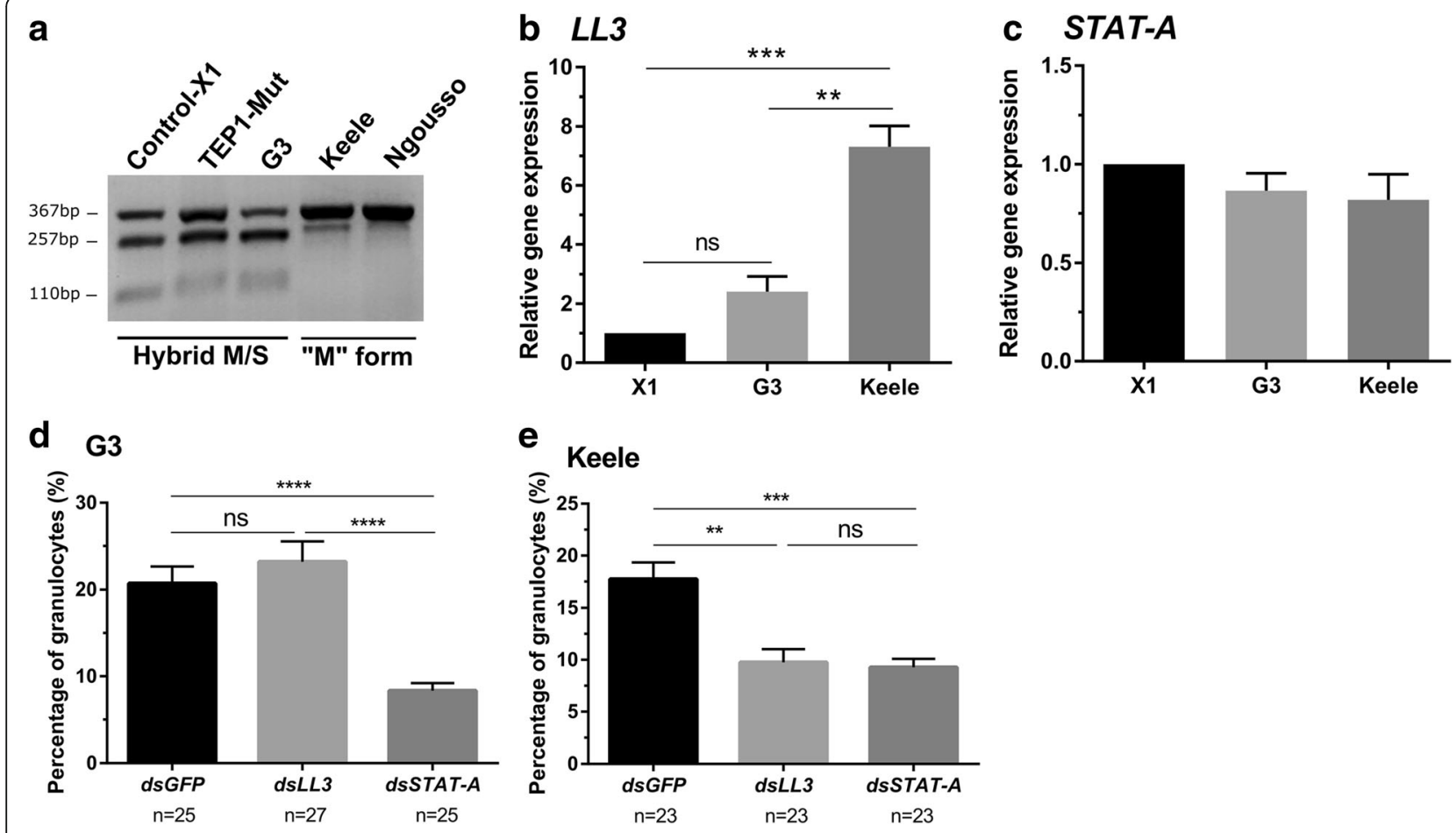

Fig. 4 Expression of $L L 3$ varies among different molecular forms of An. gambiae. A PCR-RFLP was performed to identify the molecular forms ( $M$ and S) that distinguish An. gambiae strains used in this study (X1, TEP1 mutant, G3, and Keele strains) as well as a known M-form strain (Ngousso) of An. coluzzii. DNA was prepared from individual mosquito samples for each strain and displayed consistent RFLP patterns for all mosquitoes analyzed. Individuals from each strain were used to create a representative image of the RFLP patterns for each mosquito strain (a). The X1, TEP1 mutant, and G3 strains display a hybrid pattern (M/S) of three bands (367, 257 and 110 bp), while the Keele and Ngousso strains are M molecular form (367 bp). Relative LL3 expression in the Keele strain is higher than those of the X1 and G3 strains at $24 \mathrm{~h}$ P. berghei infection (b), while there is no difference of STAT-A expression among mosquito strains (c). To examine the effects of gene-silencing on hemocyte differentiation, the proportion of granulocytes (out of total cell population) was examined in individual mosquitoes 4 days post-infection with P. berghei. The percentage of granulocytes were measured in dsGFP-, dsLL3-, or dsSTAT-A-treated mosquitoes in both the G3 (d) and Keele (e) strains. STAT-A-silencing abrogated hemocyte differentiation in both G3 and Keele mosquitoes (d) and (e), whereas LL3-silencing only influenced the Keele strain (e). Gene expression data were analyzed with a one-way ANOVA and Tukey post-hoc test, while granulocyte percentages were evaluated by Kruskal-Wallis with a Dunn's post-hoc test. Asterisks denote significance $\left({ }^{*} P<0.05,{ }^{* *} P<0.01,{ }^{* * *} P<0.001\right)$; Abbreviation: ns, not significant. Three independent biological experiments were performed for all experiments

evidence that late-phase immune responses are independent of TEP1 function. In comparisons of oocyst numbers in control and mutant lines, oocyst survival was significantly reduced in the period between day 2 and day 8 post-infection, suggesting that the loss of TEP1 does not interfere with the mechanisms of oocyst killing. Importantly, these effects were impaired when STAT-A, a known component of the late-phase immune response $[9,10]$, was silenced in the TEP1 mutant background. Together, these results argue that TEP1, and likely other immune components that target invading ookinetes, are functionally distinct from the mechanisms that influence oocyst survival.

In the course of similar gene-silencing experiments for $L L 3$, we uncovered that LL3 did not influence infection intensity or oocyst survival in the TEP1 mutant background. While in direct contrast with previously published reports $[10,17]$, these experiments were performed in the Keele strain of An. gambiae, different from the G3-derived background of the mutant TEP1 line. However, with the ability to reconstitute the effects of $L L 3$-silenicing in the Keele strain under similar insectary conditions and previous double silencing experiments for $L L 3$ and TEP1 in the Keele background [10], these experiments argue that the contrasting roles for LL3 are likely attributed to differential immune signaling amongst strains of An. gambiae.

This is supported by additional experiments examining the rDNA locus on the $\mathrm{X}$ chromosome that defines the M- and S-forms of An. gambiae (s.s.), confirming that the G3-derived strains (hybrid M/S) used in our analysis differ from that of the Keele strain ( $\mathrm{M}$ form). These genetic differences have recently led to the divergence of the $\mathrm{M}$-form as a separate species, An. coluzzii, although recent reports have argued that the Keele strain still be referred to as An. gambiae [24]. Independent of the species classifications, the differential expression of $L L 3$ between the hybrid M/S G3-derived lines and the Keele strain, but not that of STAT-A, provides strong support 
that different genetic backgrounds influence $L L 3$ gene expression with significantly effects on mosquito immune responses. Interestingly, phenotypic differences in oocyst numbers have also been described for SRPN6 [26], a known downstream target of LL3 [17], across different mosquito strains and species. Therefore, our results support the need for further study to better define the Keele strain as either An. gambiae or An. coluzzii.

Of interest, genetic polymorphisms in anti-Plasmodium immune genes have previously been described for the M- and S- molecular forms of An. gambiae/An. coluzzii across regions of Africa [27, 28]. For instance, highly allelic polymorphisms in APL1 and TEP1 genes were found in $\mathrm{S}$ form, that then underwent selective pressure towards fixation in the $M$ form $[27,28]$. Similar results of adaptive evolution in An. coluzzii for several immune genes have suggested that novel or lineagespecific immune mechanisms may have developed in the An. gambiae species complex [29]. These findings led us to examine allelic polymorphisms of LL3 and STAT-A between $\mathrm{M}$ and $\mathrm{S}$ form of An. gambiae through in silico analysis, but no obvious genetic variations in the open reading frame were detected between the molecular forms. We therefore postulate that the phenotypic plasticity of LL3 between G3-derived lines and the Keele strain might be result of epigenetic mechanisms [30], or as a result of genetic heterogeneity on yet undefined upstream factors essential for $L L 3$ regulation. However, further studies are required to comprehend the underlying molecular mechanisms regulating gene expression and subsequent immune responses between the molecular forms of the An. gambiae species complex.

Previous studies have demonstrated that there are differences in parasite recognition and killing across multiple vector-parasite combinations [3, 31-35], that at least for the human malaria parasite, P. falciparum, appear to be driven by co-evolution of the parasite with its sympatric vector [3, 34, 35]. Additional evidence argues that compatible parasites can evade immune detection $[4,36]$, supporting the idea that Plasmodium parasites in a given mosquito species can either suppress or initiate mosquito immune responses that greatly impact parasite survival. However, previous experiments have shown that both $P$. berghei and P. falciparum oocysts are susceptible to late-phase immune responses in the Keele strain [10], arguing that the mechanisms of oocyst killing are conserved across Plasmodium species and are likely not mediated by differences in parasite surface molecules. Therefore, further studies are required to better understand the potential differences in late-phase signaling between the G3 and Keele mosquito lines, as well as different Anopheles species. In summary, these findings raise additional questions into the differences in immune signaling across mosquito strains and highlight the complexity of immune responses that shape mosquito vector competence to malaria parasites.

Several studies have established that hemocytes are integral to anti-Plasmodium immune responses [10, 22, 25, 37-41], however the mechanisms by which hemocytes limit parasite infection are less understood. Recent evidence argues that cellular immune responses mediated by hemocyte-derived microvesicles direct mosquito complement activation on invading ookinetes [39], yet the manner in which hemocytes influence oocyst survival has not been described. Building from our previous results [10], these data provide further support that hemocytes are key mediators of the late-phase immune response and establish that these responses are independent of TEP1 function. With these new findings showing variation between mosquito strains in the components that shape late-phase immunity, future directions will invariably have to examine how LL3, STAT-A, and possibly other components influence hemocyte differentiation in multiple mosquito vectors. Together, these findings represent a significant advancement in our understanding of late-phase immunity while highlighting future challenges to establish how hemocytes influence oocyst survival.

\section{Conclusions}

We provide evidence using a TEP1 mutant line that the absence of TEP1 does not interfere with late-phase immune responses limiting oocyst survival. Taken together with previous results [10], we provide strong evidence that components of anti-Plasmodium immune responses targeting the ookinete and oocysts stages are independent, with TEP1 and complement-mediated responses acting only on invading ookinetes. In the course of experiments, we also identified that functional heterogeneity of known late-phase immune components exists among laboratory colonies of different molecular forms, arguing that the mechanisms that influence oocyst survival may have diverged across the An. gambiae species complex.

\section{Additional files}

\footnotetext{
Additional file 1: Table S1. List of primers for dsRNA production and qRT-PCR analysis. (DOCX $14 \mathrm{~kb}$ )

Additional file 2: Figure S1. Efficiency of dsRNA knockdowns in the TEP1 mutant line. Relative quantification of STAT-A (a) and LL3 (b) transcript in the TEP1 mutant line day 2 post-injection of dsRNA. Bar represents mean \pm SEM of three independent replicates. Data were analyzed by unpaired t-test. Asterisk denotes significant difference ( $\left.{ }^{*} P<0.01\right)$ (PNG $184 \mathrm{~kb}$ )

Additional file 3: Figure S2. Basal gene expression levels of $L L 3$ and STAT-A across mosquito strains. The relative gene expression of LL3 (a) and STAT-A (b) was measured in the midgut of naïve mosquitoes from the X1, G3, and Keele strains. Additional comparisons of transcript levels from naïve and $P$. berghei-infected midguts in the $X 1, G 3$, and Keele lines were measured to examine immune activation of LL3 (c) and STAT-A (d) in response to parasite infection. Relative gene expression is displayed as the mean \pm SEM of three independent replicates. Data were analyzed with a one-way ANOVA and Tukey post-hoc test or an unpaired $t$-test. Asterisk denotes significant difference ( ${ }^{*} P<0.05,{ }^{* *} P<0.01$ ) (PNG 593 kb)
} 


\section{Acknowledgments}

This work would not have been possible without Eric Marois for graciously providing the TEP1 mutant and X1 parental mosquito lines that were integral to performing these studies. We would also like to thanks Robert Harrell of the University of Maryland Insect Transformation Facility for providing DNA samples for the Ngousso strain of An. coluzzii, and to Brendan Dunphy for providing insectary support for the mosquitoes used in this study.

\section{Funding}

This work was supported by the Agricultural Experiment Station at lowa State University and the USDA National Institute of Food and Agriculture, Hatch project $1,010,716$, to RCS.

\section{Availability of data and materials}

All data generated or analyzed during this study are included in this published article and its additional files.

\section{Authors' contributions}

HK and RCS conceived and designed research. HK and BRA performed the experiments. HK and RCS analyzed data. HK and RCS wrote the paper. All authors read and approved the final manuscript.

\section{Ethics approval and consent to participate}

This project was performed in accordance with the recommendations of the Guide for the Care and Use of Laboratory Animals of the National Institutes of Health. All experiments were carried out under the approval and supervision of the Institutional Animal Care and Use Committee (protocol 9-15-8083-M) and the Institutional Biosafety Committee (protocol 15-D/I-0008-A) at lowa State University.

\section{Consent for publication}

Not applicable.

\section{Competing interests}

The authors declare that they have no competing interests.

\section{Publisher's Note}

Springer Nature remains neutral with regard to jurisdictional claims in published maps and institutional affiliations.

Received: 29 March 2017 Accepted: 25 July 2017

Published online: 01 August 2017

\section{References}

1. WHO. World malaria report 2015. http://www.who.int/malaria/publications/ world-malaria-report-2015/en/.

2. Smith RC, Vega-Rodríguez J, Jacobs-Lorena M. The Plasmodium bottleneck: malaria parasite losses in the mosquito vector. Mem Inst Oswaldo Cruz. 2014;109:644-61.

3. Molina-Cruz A, Canepa GE, Kamath N, Pavlovic NV, Mu J, Ramphul UN, et al. Plasmodium evasion of mosquito immunity and global malaria transmission: the lock-and-key theory. Proc Natl Acad Sci USA. 2015;112:15178-83.

4. Ramphul UN, Garver LS, Molina-Cruz A, Canepa GE, Barillas-Mury C. Plasmodium falciparum evades mosquito immunity by disrupting JNKmediated apoptosis of invaded midgut cells. Proc Natl Acad Sci USA. 2015;112:1273-80.

5. Smith RC, Barillas-Mury C. Plasmodium oocysts: overlooked targets of mosquito immunity. Trends Parasitol. 2016:32:979-90.

6. Blandin S, Shiao SH, Moita LF, Janse CJ, Waters AP, Kafatos FC, et al. Complement-like protein TEP1 is a determinant of vectorial capacity in the malaria vector Anopheles gambiae. Cell. 2004;116:661-70.

7. Povelones M, Waterhouse RM, Kafatos FC, Christophides GK. Leucine-rich repeat protein complex activates mosquito complement in defense against Plasmodium parasites. Science. 2009;324:258-61.

8. Fraiture M, Baxter RHG, Steinert S, Chelliah Y, Frolet C, Quispe-Tintaya W, et al. Two mosquito LRR proteins function as complement control factors in the TEP1-mediated killing of Plasmodium. Cell Host Microbe. 2009:5:273-84.

9. Gupta L, Molina-Cruz A, Kumar S, Rodrigues J, Dixit R, Zamora RE, et al. The STAT pathway mediates late-phase immunity against Plasmodium in the mosquito Anopheles gambiae. Cell Host Microbe. 2009;5:498-507.
10. Smith RC, Barillas-Mury C, Jacobs-Lorena M. Hemocyte differentiation mediates the mosquito late-phase immune response against Plasmodium in Anopheles gambiae. Proc Natl Acad Sci USA. 2015;112:E3412-20.

11. Oliveira GA. Lieberman J, barillas-Mury C. Epithelial nitration by a peroxidase /NOX5 system mediates mosquito antiplasmodial immunity. Science. 2012:335:856-9.

12. Levashina EA, Moita LF, Blandin S, Vriend G, Lagueux M, Kafatos FC. Conserved role of a complement-like protein in phagocytosis revealed by dsRNA knockout in cultured cells of the mosquito, Anopheles gambiae. Cell. 2001;104:709-18.

13. Povelones M, Bhagavatula L, Yassine $H$, Tan LA, Upton LM, Osta MA, et al. The CLIP-domain serine protease homolog SPCLIP1 regulates complement recruitment to microbial surfaces in the malaria mosquito Anopheles gambiae. PLoS Pathog. 2013;9:e1003623.

14. Povelones M, Osta MA, Christophides GK. The complement system of malaria vector mosquitoes. In: Raikhel AS, editor. Advances in Insect Physiology. Academic Press; 2016. p. 223-242.

15. Hurd H, Taylor PJ, Adams D, Underhill A, Eggleston P. Evaluating the costs of mosquito resistance to malaria parasites. Evolution. 2005;59:2560-72.

16. Smidler AL, Terenzi O, Soichot J, Levashina EA, Marois E. Targeted mutagenesis in the malaria mosquito using TALE nucleases. PLoS One. 2013;8:1-9.

17. Smith RC, Eappen AG, Radtke AJ, Jacobs-Lorena M. Regulation of antiPlasmodium immunity by a LITAF-like transcription factor in the malaria vector Anopheles gambiae. PLoS Pathog. 2012;8:e1002965.

18. Livak KJ, Schmittgen TD. Analysis of relative gene expression data using real-time quantitative PCR and the 2(-Delta Delta C(T)) method. Methods. 2001;25:402-8.

19. Coetzee M, Hunt R, Wilkerson R, Della Torre A, Coulibaly M, Besansky N. Anopheles coluzzii and Anopheles amharicus, new members of the Anopheles gambiae complex. Zootaxa. 2013;3619:246-74.

20. Post RJ, Flook PK, Millest AL. Methods for the preservation of insects for DNA studies. Biochem Syst Ecol. 1993;21:85-92.

21. Fanello C, Santolamazza F, Della TA. Simultaneous identification of species and molecular forms of the Anopheles gambiae complex by PCR-RFLP. Med Vet Entomol. 2002;16:461-4.

22. Smith RC, King JG, Tao D, Tomescu O, Brando C, Thallinger G, et al. Molecular profiling of phagocytic immune cells in Anopheles gambiae reveals integral roles for hemocytes in mosquito innate immunity. Mol Cell Proteomics. 2016;15:3373-87.

23. Goulielmaki E, Sidén-Kiamos I, Loukeris TG. Functional characterization of Anopheles Matrix metalloprotease 1 reveals its agonistic role during sporogonic development of malaria parasites. Infect Immun. 2014;82: 4865-77.

24. Ranford-Cartwright LC, McGeechan S, Inch D, Smart G, Richterová L, Mwangi JM. Characterisation of species and diversity of Anopheles gambiae Keele Colony. PLoS One. 2016;11:e0168999.

25. Ramirez JL, Garver LS, Brayner FA, Alves LC, Rodrigues J, Molina-Cruz A, et al. The role of hemocytes in Anopheles gambiae antiplasmodial immunity. J Innate Immun. 2014;6:119-28.

26. Abraham EG, Pinto SB, Ghosh A, Vanlandingham DL, Budd A, Higgs S, et al. An immune-responsive serpin, SRPN6, mediates mosquito defense against malaria parasites. Proc Natl Acad Sci USA. 2005:102:16327-32.

27. Rottschaefer SM, Riehle MM, Coulibaly B, Sacko M, Niaré O, Morlais I, et al. Exceptional diversity, maintenance of polymorphism, and recent directional selection on the APL1 malaria resistance genes of Anopheles gambiae. PLoS Biol. 2011;9:e1000600.

28. White BJ, Lawniczak MKN, Cheng C, Coulibaly MB, Wilson MD, Sagnon $\mathrm{N}$, et al. Adaptive divergence between incipient species of Anopheles gambiae increases resistance to Plasmodium. Proc Natl Acad Sci USA. 2011;108:244-9.

29. Rottschaefer SM, Crawford JE, Riehle MM, Guelbeogo WM, Gneme A, Sagnon N, et al. Population genetics of Anopheles coluzzii immune pathways and genes. G3. 2014;5(3):329-39.

30. Mukherjee K, Twyman RM, Vilcinskas A. Insects as models to study the epigenetic basis of disease. Prog Biophys Mol Biol. 2015;118:69-78.

31. Collins FH, Sakai RK, Vernick KD, Paskewitz S, Seeley DC, Miller LH, et al. Genetic selection of a Plasmodium-refractory strain of the malaria vector Anopheles gambiae. Science. 1986;234:607-10.

32. Michel K, Suwanchaichinda C, Morlais L, Lambrechts L, Cohuet A, AwonoAmbene PH, et al. Increased melanizing activity in Anopheles gambiae does 
not affect development of Plasmodium falciparum. Proc Natl Acad Sci USA. 2006;103:16858-63.

33. Jaramillo-Gutierrez G, Rodrigues J, Ndikuyeze G, Povelones M, Molina-Cruz A, Barillas-Mury C. Mosquito immune responses and compatibility between Plasmodium parasites and anopheline mosquitoes. BMC Microbiol. 2009;11:1-11.

34. Molina-Cruz A, DeJong RJ, Ortega C, Haile A, Abban E, Rodrigues J, et al. Some strains of Plasmodium falciparum, a human malaria parasite, evade the complement-like system of Anopheles gambiae mosquitoes. Proc Natl Acad Sci USA. 2012;109:E1957-62.

35. Molina-Cruz A, Garver LS, Alabaster A, Bangiolo L, Haile A, Winikor J, et al. The human malaria parasite Pfs 47 gene mediates evasion of the mosquito immune system. Science. 2013;340:984-7.

36. Smith RC, Jacobs-Lorena M. Malaria parasite Pfs47 disrupts JNK signaling to escape mosquito immunity. Proc Natl Acad Sci USA. 2015;112:1250-1.

37. Rodrigues J, Brayner FA, Alves LC, Dixit R, Barillas-Mury C. Hemocyte differentiation mediates innate immune memory in Anopheles gambiae mosquitoes. Science. 2010;329:1353-6.

38. Ramirez JL, de Almeida OG, Calvo E, Dalli J, Colas RA, Serhan CN, et al. A mosquito lipoxin/lipocalin complex mediates innate immune priming in Anopheles gambiae. Nat Commun. 2015;6:7403.

39. Castillo JC, Beatriz A, Ferreira B, Trisnadi N, Barillas-Mury C. Activation of mosquito complement antiplasmodial response requires cellular immunity. Sci Immunol. 2017;2:eaal1505.

40. Pinto SB, Lombardo F, Koutsos AC, Waterhouse RM, McKay K, An C, et al. Discovery of Plasmodium modulators by genome-wide analysis of circulating hemocytes in Anopheles gambiae. Proc Natl Acad Sci USA. 2009; 106:21270-5.

41. Lombardo F, Ghani Y, Kafatos FC, Christophides GK. Comprehensive genetic dissection of the hemocyte immune response in the malaria mosquito Anopheles gambiae. PLoS Pathog. 2013;9:e1003145.

\section{Submit your next manuscript to BioMed Central and we will help you at every step:}

- We accept pre-submission inquiries

- Our selector tool helps you to find the most relevant journal

- We provide round the clock customer support

- Convenient online submission

- Thorough peer review

- Inclusion in PubMed and all major indexing services

- Maximum visibility for your research

Submit your manuscript at www.biomedcentral.com/submit

) Biomed Central 